\title{
Physico-chemical model and computer simulations of silicon nanowire growth
}

\author{
Aleksey Efremov ${ }^{1}$, Alla Klimovskaya ${ }^{1}$, Ted Kamins ${ }^{2}$, Bela Shanina ${ }^{1}$, \\ Kostyantyn Grygoryev ${ }^{1}$, Sergey Lukyanets ${ }^{3}$ \\ ${ }^{I}$ V. Lashkaryov Institute of Semiconductor Physics, NAS of Ukraine, 45, prospect Nauky, 03028 Kyiv, Ukraine \\ ${ }^{2}$ Quantum Science Research, Hewlett-Packard Laboratories, Palo Alto, CA 94304, USA \\ ${ }^{3}$ Institute of Physics, NAS of Ukraine, 46, prospect Nauky, 03028 Kyiv, Ukraine
}

\begin{abstract}
A model of catalytically enhanced CVD growth of a silicon nanowire assembly on a substrate is developed, and growth process is simulated. Thermodynamickinetic theory is used for modeling of molecular transport in the gas phase, processes near catalyst surface and nanowire side of variable curvature, bulk diffusion of silicon adatoms through catalyst - body, and 2D nucleation. The simulation of atomic transport across surfaces is based on a long-wave approach of lattice gas approximation. To determine a character of atomic transport in $\mathrm{TiSi}_{2}$-catalyst that is of great importance for application in Si-based technology, a density functional theory is used. The main result of modeling is that it is found a relationship between growth conditions (an initial radius of catalyst particles, their density, substrate temperature, content, pressure of gas, as well as properties of materials used) and, on the other hand, a growth rate, shape, composition, and type of atomic structure (amorphous or crystalline) of the nanowires grown. Besides, available experimental data published previously are discussed, and a qualitative agreement between theory and various experiments is obtained. This agreement gives rise to use the found relationship for controlling the nanowire growth.
\end{abstract}

Keywords: silicon, nanowire.

Manuscript received 05.05.05; accepted for publication 25.10.05.

\section{Introduction}

Silicon-based nanotechnology is highly promising because of its compatibility with conventional silicon integrated technology [1-3]. Varying the experimental conditions allows to achieve a wide variety of nanowire shapes [4], such as straight nearly circular right cylinders or prisms, telescope-like objects, cones, bead-like freestanding filaments, springs $[5,6]$ or even single spheres [7]. Very often we do not know why a specific shape is realized by a given process, or why the shape changes, although all the process parameters appear to be properly controlled. The wide range of realized shapes makes it difficult to expect that any model based on chemical kinetics or/and thermodynamics can successfully predict or explain such complicated and varied behavior. Some doubts also arise as to the applicability of these macroscopic tools to nanoscale dimensions. Nevertheless, recently obtained results have shown [8] that considerations based on the approaches mentioned above can predict some aspects of the experimental behavior, especially the shape transformation of the nanoobject.
This paper is devoted to the detailed theoretical analysis of the mechanisms responsible for quasi-1D nanocrystal growth by catalytically enhanced CVD technique that includes multiphase transformations in a multicomponent medium. We consequently use concepts of driving forces, barriers, and resistances toward atomic or molecular transport; used together these concepts determine the transfer of building materials along different routes in series or in parallel towards the places where atoms are incorporated into the growing object and by-products are removed from the reaction zone. The atomic transport to a solid phase and surfaces was simulated as atomic exchange between adjacent small cells of the surface. For a practically important case (1D growth of silicon with the use of crystalline titanium disilicide C49 as a catalyst), the distribution of electron density, potential relief, and activation energy for diffusion have been calculated for different crystallographic planes of the catalyst using the density functional method. This made it possible to specify mechanisms of diffusion. 
The paper is organized as follows. Section 2 presents a basis of physical model and contents: a review of physical processes included in the model (subsection 2.1), mathematical description of gas transport of silane (subsection 2.1.1), temperature distribution in the gas phase near the substrate, chemisorption stage (subsection 2.1.2), diffusion processes on the surface, bulk and interface of the catalyst particle (subsection 2.1.3) and nanowire side (subsection 2.1.4). Mathematical formulation of the model is presented in 2.2 and results are discussed in Section 3.

\section{Physical model}

\subsection{Review of physical processes included in the model}

In contrast to the growth models proposed earlier $[4,9$, $10]$, which were forced to be more or less schematic, we have tried to take into account all the important stages of the nanowire growth process.

To simulate the growth, we will restrict ourselves to the consideration of silicon nanowire growth on a silicon substrate by using silane in a mixture with hydrogen $\left(\mathrm{SiH}_{4}+\mathrm{H}_{2}\right)$. Transition to different gaseous mixtures will not lead to qualitatively new processes although it will affect the growth quantitatively. As a catalyst, any of metals used in this process may be considered (e.g., Au, $\mathrm{Ni}, \mathrm{Pt}, \mathrm{Pd}, \mathrm{Ga}, \mathrm{Cu}$ ) or chemical compounds being in liquid, solid or even crystalline state during crystal growth. Catalytic particles are supposed to be previously formed on the silicon substrate. Hereafter, they are assumed to be hemispheres with the radius $R$.

Simulations included two groups of the processes, which are treated in different manners. The first group concerns the gas-phase transport of $\mathrm{SiH}_{4}$ molecules to the vicinity both of the catalyst particle, and towards the side of the nanowire. The second group involves various processes on solid phase surface, at catalyst/nanowire interface, and in the bulk of catalyst. General view of the growing nanowire and fluxes responsible for mass transfer in the vicinity of the nanowire and on the surface of the object are shown in Fig. 1. In the present approach, the mass-transfer in gas phase is considered as a steady-state macroscopic flux caused by chemical potential difference and determined by respective "resistances" and barriers at different stages of the gasphase delivery. Therefore, here we consider only the nanowire growth, corresponding to the steady-state gasphase conditions. On the other hand, the mass-transfer onto the surface or into the bulk of a growing object is considered in the framework of an approach similar to a lattice-gas approximation (Fig. 1). Namely, the catalyst surface, catalyst/nanowire interface, and nanowire sidewall are divided into discrete cells, exchanging with each other by silicon or hydrogen adatoms, and undergoing also the adsorption and desorption of gasphase molecules.

\subsubsection{Gas phase}

In the steady-state mode, the gas phase may be subdivided into five zones (numeration is shown in Fig. 1):

1. Forced convection region, blown by the gas mixture, where the input steady-state values of concentrations of both gases are established.

2. Macroscopic diffusion layer, characterized by normal gradient of active gas concentration as well as normal gradient of temperature. The former is caused by the joint active gas consumption by the whole aggregate of the catalyst particles as well as (if any) by the sidewalls of the growing nanowires.

3. The region directly adjacent to the ensemble of growing wires and depleted by silane because of silane decomposition on the ensemble.

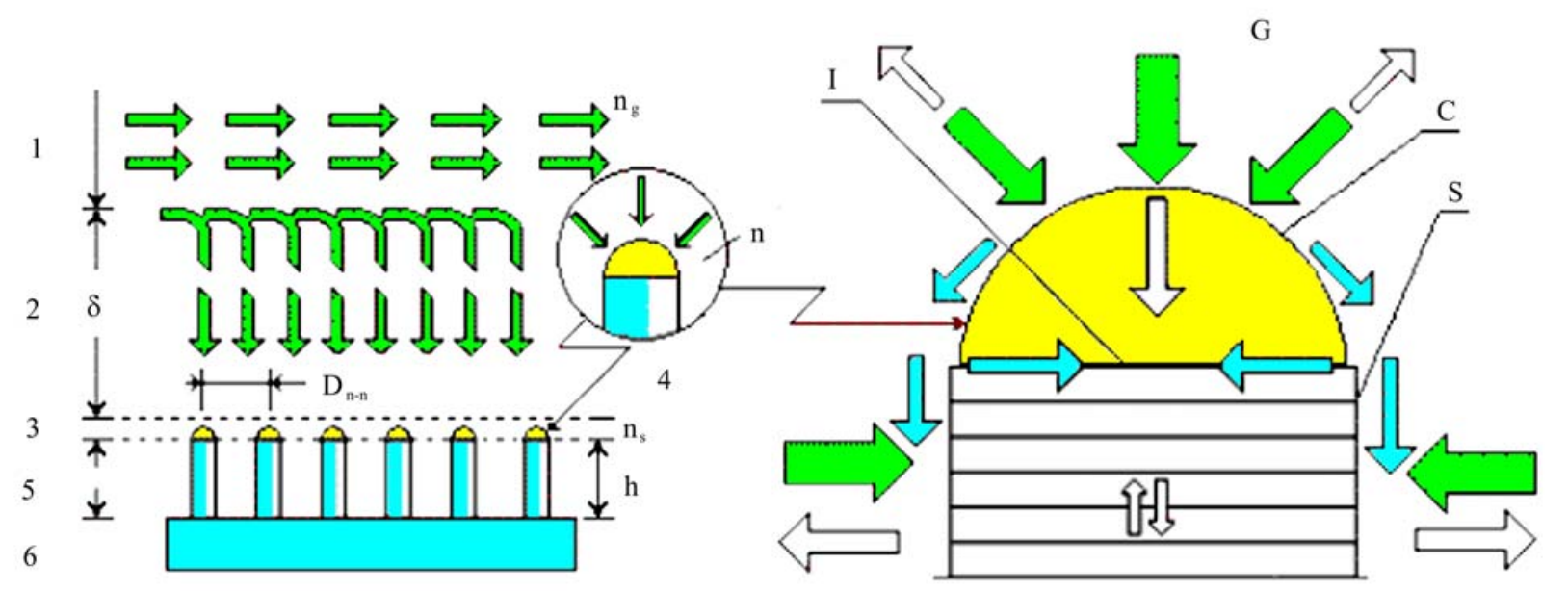

Fig. 1. General view of interaction of gas phase with the ensemble of growing nanowires (arbitrary scale) and distribution of fluxes of substance considered in the model of individual nanowire growth. Arrows indicate macroscopic distribution of active gas or adatom fluxes. 1-5 are zones of gas phase listed in the text; $h$ and $D_{n-n}$ are an average height and distance between neighboring nanowires, respectively, 6 is the substrate. Letters corresponds to considered parts of the nanowire: external surface of a catalyst particle (C), interface (I), sidewall (S), nearest vicinity of the gas phase (G).

\section{(C) 2005, V. Lashkaryov Institute of Semiconductor Physics, National Academy of Sciences of Ukraine}


4. Local vicinity of the individual catalyst particle.

5. Inside the nanowire ensemble.

The total effective steady-state flux which is absorbed by the individual growing nanowire through the external surface of catalyst can be written as

$J_{a}=k_{\mathrm{eff}}\left(n_{g}-n_{\mathrm{eq}}\right)$,

where $k_{\text {eff }}\left(k_{\text {eff }}^{-1}=k_{D}^{-1}+k_{R}^{-1}\right)$ is the effective rate coefficient, which includes both diffusive transport of reactant gas through the boundary diffusion layer and the silane decomposition reaction. The similar approach was used for CVD of silicon in [19]. Here $n_{g}$ is the active gas concentration in the first zone, $n_{\mathrm{eq}}$ is an equilibrium concentration near the solid surface. It is modified by the Gibbs-Thomson effect [17, 18] accounted usually in precipitate growth consideration and used for explanations of microwire growth [4]:

$n_{\mathrm{eq}}=n_{\mathrm{eq} 0} \exp \left(\gamma_{m} \Omega_{m} K_{m} / k_{\mathrm{B}} T\right) \approx$

$\approx n_{\mathrm{eq} 0}\left(1+\gamma_{m} \Omega_{m} K_{m} / k_{\mathrm{B}} T\right)$,

where the average curvature $K_{c}=2 / R$ for the hemispherical surface of the catalyst nanoparticle and $K_{s}=1 / R$ for the side of the cylindrical nanowire, $\Omega_{m}$ is the atomic volume of silicon, and $\gamma_{m}$ is the specific surface free energy for the catalyst $(m=c$ ) or for silicon $(m=s), n_{\text {eq } 0}$ is the equilibrium concentration for the corresponding flat surface, $T$ is the gas temperature near the surface of catalyst. Using the reaction rate theory and mass-balance considerations [16-18] the diffusion and reaction rate coefficients for our case may be obtained in the form:

$k_{R}=\left(1-\Theta_{c}-\Phi_{c}-\Phi_{2 c}\right) \cdot \sqrt{n_{s} / n_{\mathrm{eq} 0}} \cdot v_{0} \times$

$\times \exp \left(-\frac{\gamma_{c} \Omega_{c} K_{c}}{2 k_{\mathrm{B}} T}\right) \cdot \exp \left(-\frac{W_{a c}}{k_{\mathrm{B}} T}\right)$.

$k_{D}=D_{M} /\left(2 \pi R^{2} \delta \cdot N_{c}\right) \equiv\left(D_{M} / \delta^{*}\right)$,

where $\Theta_{c}, \Phi_{c}$, and $\Phi_{2 c}$ are the fractional coverages of the catalyst surface by silicon and hydrogen adatoms and $\left(\mathrm{H}_{2}\right)$-complexes [11]. Here $v_{0}$ is approximately the thermal (or diffusion) velocity for an impinging silane molecule $\left(v_{0} \approx 600 \mathrm{~m} / \mathrm{s}\right), \quad W_{a c}$ is the barrier for chemisorption, $N_{c}$ is the number of catalytic particles per unit area, $n_{s}$ is the effective average silane concentration near the solid surface, $\delta=d_{\text {reactor }} / \mathrm{Nu}$ is the thickness of diffusion layer [12], $d_{\text {reactor }}$ is the characteristic size of reactor, $\mathrm{Nu}$ is the Nusselt criterion and $D_{M}$ is the molecular diffusion coefficient. The particular case of small admixture of silane in gas mixture with hydrogen is well studied both experimentally [12] and theoretically [13, 14] and $D_{M} \propto T_{g}^{1.75} P_{\text {total }}^{-1}$, where $T_{g}$ is the temperature of gas. The approximate formulas that we used in calculations $[13,14]$ give the similar results (difference did not exceed $20 \%$ ). The input gas mixture temperature was usually close to the room one, and the substrate was maintained at the growth temperature $T_{s}>500{ }^{\circ} \mathrm{C}$. Therefore, there was a temperature gradient in the direction perpendicular to the substrate surface. According to direct experimental data [19], the thickness of the region with variable temperature (thermal layer [11]) may be close to the thickness $\delta$ of layer in the CVD reactor. In this case, the thermodiffusion will result in a small $(\approx 15 \%)$ additional counter flux of silane molecules directed from the hot substrate to the bulk of gas phase $[12,14]$. On the other hand, the diffusion rate constant $k_{D}$ depending on $D_{M}$ should be averaged over the temperature inside the diffusion layer [12].

Numerical estimations show that for $P_{\text {total }}=10$ Torr, $\delta=5 \mathrm{~cm}, \quad R=10^{-6} \mathrm{~cm}, \quad N_{c}=10^{7} \mathrm{~cm}^{-2} \quad$ we have $D_{M} \approx 50 \mathrm{~cm}^{2} / \mathrm{s}, \delta^{*} \approx 3 \mu \mathrm{m}>R, k_{D} \approx 2 \cdot 10^{5} \mathrm{~cm} / \mathrm{s}$. For $k_{R}$ at $T=640^{\circ} \mathrm{C}$ and $\Delta E_{a c}=W_{a c}+\gamma_{c} \Omega_{c} K_{c} / 2 \approx$ $\approx 0.5 \mathrm{eV}$ we have the value about of $200 \mathrm{~cm} / \mathrm{s}$. Only for dense ensembles the diffusion delivery can limit the process and detailed consideration of thermodiffusion is wath-while.

Mass-transfer inside the nanowire ensemble qualitatively depends on the ensemble density. For quasi-isolated nanowires the silane concentration in the vicinity of sidewall is the same as in the bulk gas phase. For the moderate density of the wires on the substrate when $D_{n-n}$ (Fig. 1) is about several free paths of asilane molecule $\lambda_{m}$, the mass-transfer may be treated yet as molecular diffusion. However, the average concentration of active gas inside the ensemble is determined by cooperative consumption of silane by a group of nearest neighbors. The steady-state flux on the sidewalls may be described by relation (1) with $n_{g}$ replaced with $n_{g}^{\text {eff }}$. In this case, $k_{R}$ is described by the formula (3) with $W_{a c} \approx 1.5 \mathrm{eV}$. Using considerations similar to those in [18] for ensemble of simultaneously growing precipitates, we may obtain the expression for sidewall of cylindrical nanowires:

$k_{D}^{s}=\frac{D_{M}}{R \cdot \ln \left(D_{n-n} / 2 R\right)} \quad$ and $\quad k_{\text {eff }}^{s^{-1}}=k_{D}^{s^{-1}}+k_{R}^{-1}$.

At the high density of the wires when $D_{n-n} \leq \lambda_{m}$, the flux on sidewalls should be considered as Knudsen's one $[13,14]$ and the precise expression of the transport coefficient depends on the peculiarities of the inside arrangement of the ensemble. Direct Monte-Carlo simulations of molecular trajectories would be 
worthwhile here [15]. We will consider this problem in detail in another work.

\subsubsection{Processes in solid phase}

In this model, we consider the following growth-related processes in the solid phase.

(i) Single center adsorption of silane $[11,20]$ (on the catalyst or on the side surface) accompanied by release of silicon and hydrogen adatoms and subsequent desorption as molecular hydrogen (empty arrows, Fig. 1):

$$
\begin{aligned}
& \mathrm{SiH}_{4} \rightarrow \mathrm{Si}_{\mathrm{ad}}+4 \mathrm{H}_{\mathrm{ad}}, \\
& 2 \mathrm{H}_{\mathrm{ad}} \rightarrow\left(\mathrm{H}_{2}\right)_{\mathrm{ad}} \rightarrow \mathrm{H}_{2} \uparrow .
\end{aligned}
$$

Possible intermediate by-products (e.g., $\mathrm{SiH}_{3}, \mathrm{SiH}_{2}$, and $\mathrm{SiH}$ ) are not considered in the present model.

(ii) Bulk diffusion of silicon atoms towards the top of the nanowire through the catalyst particle.

(iii) Surface diffusion of adatoms across the catalyst nanoparticles to the catalyst/Si interface.

(iv) Diffusion of adatoms along the interface to form an atomic step that propagates from the periphery to the center of the nanowire.

(v) Adatom transport from the catalyst surface toward and along the side of the nanowire.

(vi) Nucleation and growth of 2D nucleus both on the sidewall and at the interface.

\subsubsection{Migration of adatoms}

In the considered problem, the two-component surface diffusion of silicon and hydrogen having different local surface mobility is of importance. In general, the effective diffusion coefficient of each component depends nonlinearly on surface site occupation by both components. Besides, at coverages near percolation limit a set of new nonlinear effects and instabilities caused by topology peculiarities of atomic arrangement, e.g., surface flux anisotropy are predicted. All of these effects are out of scope of this paper and will be considered in another work. On the surface divided into cells [20], the atomic migration (diffusion) along the surface (interface) is considered as averaged spill over from cell-to-cell with characteristic (mesoscopic) frequency of transfer $v_{m}=\alpha D_{\text {loc }} / L_{c}^{2}$, i.e., Einstein's relation adopted as valid, which is to be a case not very close to percolation threshold. Here, $D_{\mathrm{loc}}=\lambda^{2} / 4 \tau$ is the local diffusivity in the commonly used Arrhenius form, $L_{c}$ is the size of cell, and $\alpha$ is the numerical coefficient, depending on the shape of cell [12]. Local fluxes between the cells are governed by the total number of unoccupied sites in the destined cell. Exact analytical solution for $n$-component lattice gas gives corresponding multiplier as $\left(1-\theta_{1}\right)\left(1-\theta_{2}\right) \ldots\left(1-\theta_{n}\right)$ [21] for probability of a jump to the destine cell, valid however only for small coverage. For this multiplier extrapolation we adopt the approximate formula in the form $\left(1-\theta_{1}-\theta_{2} \ldots-\theta_{n}\right)$. We will check it and defined more exactly by $\mathrm{MC}$ experiments.

Respective "diffusion" terms in the balance equation for coverage with silicon $(\Theta)$ and hydrogen $\left(\Phi_{1}\right)$ adatoms for some cell are presented in the model in finite-difference form as follows:

$$
\begin{aligned}
& D_{\mathrm{Si}} \nabla^{2} \Theta \rightarrow \hat{D} \Theta= \\
& =-\Theta \cdot \sum_{m} v_{m}^{\mathrm{Si}}\left(1-\Theta_{m}-\Phi_{1 m}-\Phi_{2 m}\right)+ \\
& +\sum_{m} v_{m}^{\mathrm{Si}}\left(1-\Theta-\Phi_{1}-\Phi_{2 m}\right) \cdot \Theta_{m} \\
& D_{\mathrm{H}} \nabla^{2} \Phi_{1} \rightarrow \hat{D} \Phi_{1}= \\
& -\Phi_{1} \cdot \sum_{m} v_{m}^{\mathrm{H}}\left(1-\Theta_{m}-\Phi_{1 m}-\Phi_{2 m}\right)+ \\
& +\sum_{m} v_{m}^{\mathrm{H}}\left(1-\Theta-\Phi_{1}-\Phi_{2}\right) \cdot \Phi_{1 m}
\end{aligned}
$$

where summation is taken over nearest cells adjacent to the considered one, $\Phi_{2}$ is the coverage with immobile $\mathrm{H}_{2}$-complexes. Atomic migration along nanowire is complicated by the energetic nonequivalence of different parts of the surface, caused by varied parameter $\gamma K$ where $\gamma$ is the surface free energy and $K$ is the curvature. For nanowire with a cylindrical body and hemispherical catalyst particle, it may only result in the addition flux from catalyst surface to sidewall. However, for nanowires with complicated shape we will have an addition drift component of surface flux in the mass balance throughout the surface. It may be presented in the following form:

$J_{d r}=-\frac{D_{\mathrm{Si}} \Omega}{k_{\mathrm{B}} T_{S}} \sigma \Theta \nabla(\gamma K)$,

where $\Omega$ is the atomic volume, $\gamma K$ relates to the part of the surface, belonging to the given cell, $\sigma$ is the number of sites per init area. We will touch the problem of evolution of complicate forms in Discussion. The alternative mechanism of silicon adatoms delivery to the interface is the bulk diffusion through the catalyst particle or droplet. Therefore, we consider the models suitable for two kinds of catalysts: a gold-silicon alloy, which is liquid during the nanowire growth, and $\mathrm{TiSi}_{2}$ (C49), which is solid during growth. For the gold-silicon system, we take the diffusion of $\mathrm{Si}$ in a gold catalyst droplet to be isotropic and consider a two-step process. First, Si atoms accumulate in the bulk of the catalyst up to a (temperature-dependent) solubility $\operatorname{limit} C^{*}$; silicon atoms then precipitate from the catalyst onto the top of the nanowire and are incorporated there. 
In order to determine the character of atomic transport across the $\mathrm{TiSi}_{2}$ (C49) surface and through its bulk, we computed the electronic structure for $\mathrm{TiSi}_{2}$ (C49). Its crystalline cell is shown in Fig. 2a. The calculations were done using the full-potential linearized augmented plane-wave method based on the Kohn-ShamHohenberg density-functional theory to account for the exchange-correlation energy. A computational program package WIEN-2k [23] was used. Fig. 2b shows a twodimensional map of the electronic density in the (110) plane. This plane is the one observed on the top of the most prevailing type of $\mathrm{TiSi}_{2}$ catalyst nanoparticles, as well as the plane in contact with the (100) plane of silicon at the interface. The space between two crystallographic planes is the location of easiest diffusion because the inter-atomic bonds are weak and the electron density is very small in these spaces. Figure 2 shows that, for $\mathrm{TiSi}_{2}$, there are easy paths for direct diffusion of adatoms from the external surface through the bulk to the catalyst/silicon interface along $\langle 110\rangle$ directions. Calculations show that activation energies for diffusion of silicon through $\mathrm{TiSi}_{2}$ bulk is rather high for the vacancy mechanism $(2.72 \mathrm{eV})$, but is about $1 \mathrm{eV}$ or less for interstitial mechanism. Therefore, bulk diffusion via this mechanism may be at least competitive channel for atomic delivery to the growing interface.

\subsubsection{Interface building up}

Building up of catalyst/nanowire interface is limited not only by atomic delivery itself, but also by interfacial facet nucleation and growth [8]. The same process should be on the sidewall. In addition to bulk diffusion through the catalyst particle, we have considered migration of Si-adatoms by first diffusing along the catalyst surface and then diffusing along the catalyst/nanowire interface from its periphery to its center. Delivering $\mathrm{Si}$ atoms to the interface by bulk diffusion does not introduce any qualitatively new irregularities to the growth front; the axial growth rate is determined by the steady-state value $\Theta_{c}$. It is worth to note that the surface/interface diffusion mechanism produces a propagating step, which results in timevarying material consumption. The oscillations resulting from the layer-by-layer propagation of the step at the interface influence not only the instantaneous axial and radial growth rates, but also the concentrations of all silicon species, including the concentrations of adatoms on the catalyst and even in gas phase near the surface. Nucleation and growth for the sidewall and the interface are included in the model as follows. When the concentration of mobile atoms inside the cell exceeds some equilibrium value $\Theta_{\mathrm{eq}}$ the primary nucleation begins with the rate of solid-phase accumulation which is equal to $\beta_{n} \cdot\left(\Theta_{m}^{g}-\Theta_{\mathrm{eq}}\right)$, where $\beta_{n}$ is the nucleation rate constant, $\Theta_{m}^{g}$ is normalized concentration of mobile atoms (superscript "g") on either sidewall $(m=s)$ or interface $(m=i)$. Accounting for the Gibbs-Thomson effect resulted from curvature of the boundary between a 2D-nucleus at the interface plane and the adjacent 2Dsea of mobile atoms, we may present $\Theta_{\text {eq }}$ as:

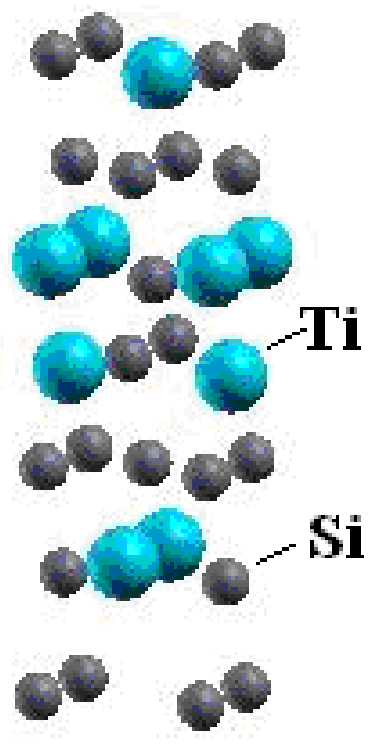

$a$

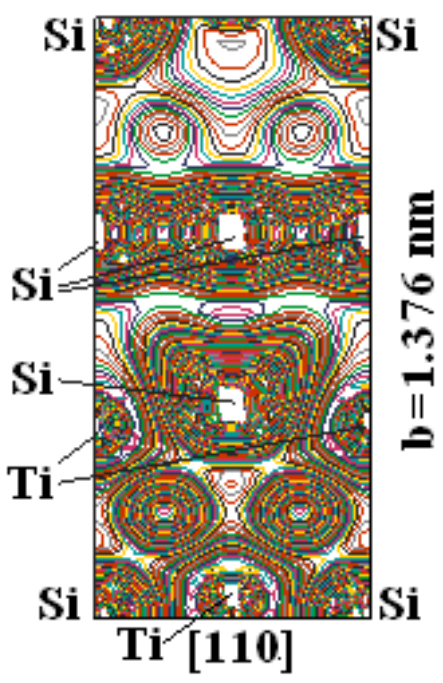

$b$

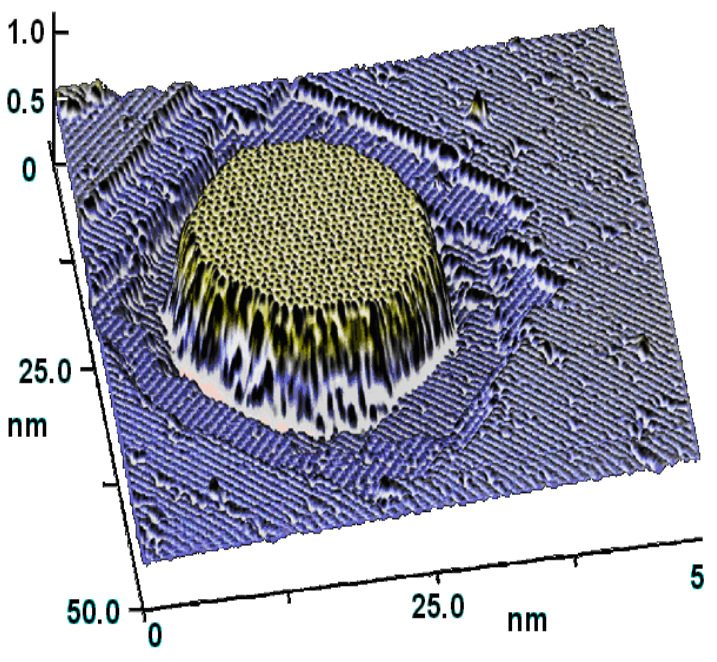

Fig. 2. Atomic arrangement at $\mathrm{TiSi}_{2}$ surface.

$a$ - Primitive cell of $\mathrm{C} 49 \mathrm{TiSi}_{2}$; the lattice parameters are: $d_{a}=3.6 \AA, d_{b}=13.76 \AA, d_{c}=3.62 \AA$. Silicon atom rows correspond to the directions of easy diffusion (b). Map of the electron density $\rho$ in the (110) plane; $\rho_{\max }=0.05$ el $/$ a.u. ${ }^{3}$ (a.u. $=0.529 \cdot 10^{-8} \mathrm{~cm}$ ); $\rho_{\min }=0.0005$ el/a.u. ${ }^{3}$. Distance between lines corresponds to $\Delta \rho=0.001$ el/a.u. ${ }^{3}$ (c) Scanning-tunneling micrograph of $\mathrm{TiSi}_{2}$ island [23]. 
$\Theta_{\text {eq }}=\Theta_{\text {eq } 0} \exp \left(\gamma_{\text {line }} \cdot \Xi \cdot K_{\text {line }} / k_{\mathrm{B}} T\right)$,

where $\Theta_{\text {eq } 0}$ is the equilibrium coverage by mobile atoms in the presence of straight line atomic step, $\gamma_{\text {line }}$ is the line tension of the germ [8], $K_{\text {line }}$ is the germ boundary curvature (for single nucleus mode $K_{\text {line }} \approx 1 / R$ ) and $\Xi=1 / \sigma_{i}$ is the effective area per one atom. 2D-solidphase, being formed at once continues to grow irreversibly according to second-order reaction between the solid germ and $2 \mathrm{D}$-gas phase. Respective term is $\beta_{g} \Theta_{m}^{g} \Theta_{m}^{s}$, where $\beta_{g}$ is the growth rate constant, superscript "s" relates to solid phase. Both for $\beta_{g}$ and

$\beta_{n}$ the simple Arrhenius form is adopted. In the model we consider the simultaneous growth of three monolayers at the interface and on sidewall. Besides the jumps of mobile atoms from upper monolayer are included in the form $\alpha \Theta_{i+1}^{g}\left(1-\Theta_{i}^{g}-\Theta_{i}^{S}\right)$. The real elongation growth rate is calculated by direct accounting for all atoms (irrespective of a delivery way) incorporating at the interface region per unit area and unit time.

\subsection{Rate equations included in the model}

Catalyst surface

$\partial \Theta_{c} / \partial t=J_{a} / \sigma_{c}-\beta \cdot \Theta_{c}+\hat{D}_{c}^{\mathrm{Si}} \Theta_{c}$

$\partial \Phi_{c} / \partial t=4 J_{a} / \sigma_{c}-2 k_{a s c} \Phi_{c}^{2}+\hat{D}_{c}^{\mathrm{H}} \Phi_{c}$

$d \Phi_{2 c} / d t=k_{a s c} \Phi_{c}^{2}-k_{d e s} \Phi_{2 c}$,

$\left.J_{c}\right|_{R}=\left.\left(k_{c i}+k_{c s}\right) \sigma_{c} \Theta_{c}\right|_{R}-\left.k_{s c} \sigma_{s} \Theta_{s}\right|_{h}-\left.k_{i c} \sigma_{i} \Theta_{i}\right|_{R}$,

sidewall (three monolayers)

$$
\begin{aligned}
& \partial \Theta_{s}^{g} / \partial t=J_{a} / \sigma_{s}-\beta_{n}^{\prime}\left(\Theta_{s}^{g}-\Theta_{0}\right)-\beta_{g}^{\prime} \Theta_{s}^{g} \cdot \Theta_{s}^{s} \\
& +\hat{D}_{s}^{\mathrm{Si}} \Theta+\alpha \Theta_{i+1}^{g}\left(1-\Theta_{i}^{g}-\Theta_{i}^{s}\right)_{s}^{g}-\alpha \Theta_{i}^{g}\left(1-\Theta_{i-1}^{g}-\Theta_{i-1}^{s}\right) \\
& \partial \Theta_{s}^{s} / \partial t=\beta_{n}^{\prime} \cdot\left(\Theta_{s}^{g}-\Theta_{0}\right)+\beta_{g}^{\prime} \cdot \Theta_{s}^{g} \cdot \Theta_{s}^{s}, \\
& \partial \Theta_{s}^{s} / \partial t=\beta_{n}^{\prime} \cdot\left(\Theta_{s}^{g}-\Theta_{0}\right)+\beta_{g}^{\prime} \cdot \Theta_{s}^{g} \cdot \Theta_{s}^{s}, \\
& \left.J_{s}\right|_{h}=\left.k_{c s} \cdot \sigma_{c} \cdot \Theta_{c}\right|_{R}-\left.k_{s c} \cdot \sigma_{s} \cdot \Theta_{s}^{g}\right|_{h},
\end{aligned}
$$

interface (three monolayers)

$$
\begin{aligned}
& \partial \Theta_{i}^{g} / \partial t=-\beta_{n} \cdot\left(\Theta_{i}^{g}-\Theta_{e q}\right)+ \\
& \hat{D}_{i}^{S i} \Theta_{i}^{g}-\beta_{g} \Theta_{i}^{g} \Theta_{i}^{s}+\alpha \Theta_{i+1}^{g}\left(1-\Theta_{i}^{g}-\Theta_{i}^{s}\right) \\
& -\alpha \Theta_{i}^{g}\left(1-\Theta_{i-1}^{g}-\Theta_{1-i}^{s}\right),
\end{aligned}
$$

$d \Theta_{i}^{s} / d t=\beta_{n}\left(\Theta_{i}^{g}-\Theta_{e q}\right)+\beta_{g} \Theta_{i}^{g} \Theta_{i}^{s}$,

$\left.J_{i}\right|_{R}=\left.k_{c i} \sigma_{c} \Theta_{c}\right|_{R}-\left.k_{i c} \sigma_{i} \Theta_{i}\right|_{R}$,

where $k_{c i}, k_{c s}, k_{s c}$, and $k_{i c}$ are the rate coefficients for different routes and directions of mass transfer ( $c \leftrightarrow s$ and $c \leftrightarrow i$ ) at the boundary, $\sigma_{c}, \sigma_{s}$ and $\sigma_{i}$ are the numbers of adsorption centers per unit area of catalyst, sidewall surface, and interface, respectively. The subscript " $R$ " corresponds to cells both at the edge of the dome-shaped catalyst and at the periphery of the nanowire top, which are in contact with each other.

\section{Results of simulation and discussion}

To demonstrate the main features of the nanowire growth, we present below results of numerical solution of the system of kinetic equations (10)-(21) written for each taken separately spatial cell on the external surfaces or at the interface (Fig. 2). A set of dependences of the elongation growth rate $v_{h}\left(R, T_{s}, P_{g}\right)$ of nanowires on radius of the nanowire, temperature of the substrate and partial pressure of silane in gas phase was calculated. Kinetics of wire growth for different mechanisms of Siatom delivering is presented in Figs 3 and 4. Here the work of the program for the growth simulation used in the present paper is demonstrated. Kinetic dependences for surface (interface) concentrations of various components are shown at the left-hand side of the window. While at the right hand side the animation of wire growth is presented.

The time evolution of three upper monolayers just below the moving interface is considered concurrently. Initial increase of the concentration of mobile silicon atoms at the interface is due to increase of Si-atom delivery through the bulk of catalyst body and around the catalyst external surface. Its following decrease is caused by the mobile atoms attachment to the nucleating

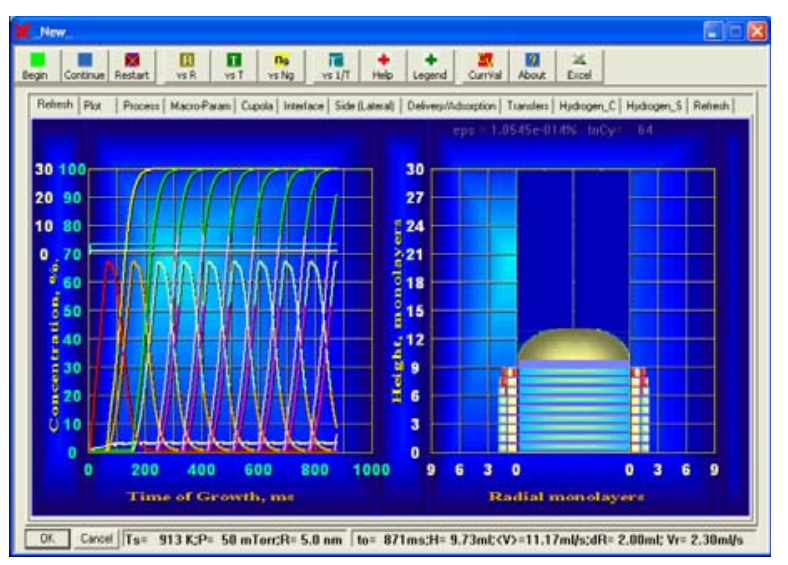

Fig. 3. Formation of near cylindrical nanowire with core-shell structure. 


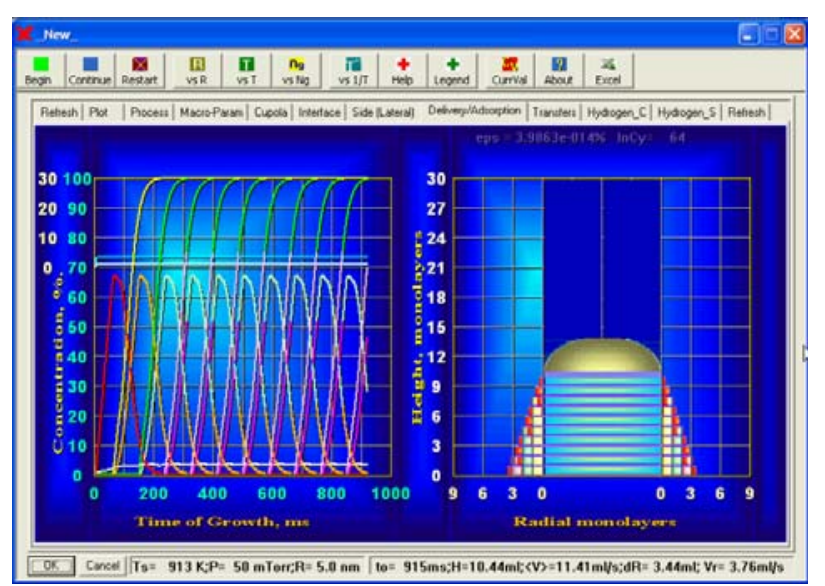

Fig. 4. Formation of wire-like heterostructure with a cone-like shell.

and growing facet. Fig. 3 shows the kinetics of nanowire growth, when diffusion of silicon adatoms across the catalyst surface, interface and sidewall supplies building blocks to the growing crystal. The nanowire presents some heterostructure consisting of a shell and core. It is worth to note that a thickness of the shell depends, in particular, on the elongation growth rate.

Fig. 4 shows the growth of the nanowire, when all the ways of silicon atom delivering including the direct silane adsorption on the sidewall are involved into the growth. This is a case of wire-like heterostructure with a cone-like shell. The degree of tapering depends here not only on relation between fluxes of substance towards the catalyst surface and to the sidewall of the nanowire, but also on details of behavior of mobile atoms upon the sidewall.

The data taken for simulation consists of microparameters presenting the used substances and macroparameters reflecting the growth conditions. To demonstrate main peculiarities of the growth, we use micro parameters that are close to those for bulk silicon, being fully aware that they may be different from those for nanoobjects. Due to the lack of the data and in order to simplify the model, we assume all the activation energies to be the same. Their exact values may be estimated only from comparison with comprehensive experiments. Nevertheless, qualitative conclusions based on the results of simulation as well as recommendations for the technologists of the way to control properties of nanowires might be made.

The following microparameters were used to receive the results presented below unless specified otherwise: the elementary atomic volume $\Omega=0.02 \mathrm{~nm}^{3}$; specific surface energy $\gamma=6.24 \mathrm{eV} / \mathrm{nm}^{2}$; density of adsorption centers (it is suggested to be equal to the density of the surface atoms) $\sigma=13.57 \mathrm{~nm}^{-2}$; pre-exponent factors for surface diffusion: across the catalyst $D_{c 0}=5 \cdot 10^{4} \mathrm{~nm}^{2} / \mathrm{ms}$, along the catalyst $/$ nanowire interface $D_{i 0}=5 \cdot 10^{4} \mathrm{~nm}^{2} / \mathrm{ms}$, across the sidewall
$D_{s 0}=5 \cdot 10^{3} \mathrm{~nm}^{2} / \mathrm{ms}$; pre-exponent factors for the bulk diffusion $D_{b 0}=2 \cdot 10^{4} \mathrm{~nm}^{2} / \mathrm{ms} ;$ activation energies $W_{a}=E_{a}=0.6 \mathrm{eV}$.

The simulations were carried out for the various growth conditions that are indicated in the captions of Figures. Instead of concentrations $n_{g}, n_{\text {eq } 0}$ of silane in gas phase and near the surface we give a partial $P_{g}$ pressure and equilibrium $P_{\text {eq } 0}$ pressure related to a flat surface. Relations between them are defined by conventional expression $n=P / k_{\mathrm{B}} T$. The total pressure of the gas mixture is supposed to be equal to 10 Torr.

Fig. 5 shows the growth rate dependences versus the wire radius that are corresponding to the simple case of nanowire growth, when only one way of $\mathrm{Si}$ atom delivery is accounted, namely, bulk diffusion through the catalyst-body (arrow 7, Fig. 1). The varied parameter for the curves is the partial pressure of silane.

This is a case when the wire grows in one direction and in the form of homogeneous solid cylinder without any shell.

To understand the mechanisms that are limiting the growth, the same results are presented in coordinates $v$ and $1 / R$ in Fig. 6 . As can be seen from this figure, the dependence of growth rate versus inverse radius have a maximum at certain wire radius $R_{\max }$ that shifts to the region of smaller values with an increase of the silane pressure. Furthermore, both the left and right shoulders of the curves indicate a linear relation between the growth rate and the inverse wire radius. The left shoulder of the curves $R \geq R_{\max }$ is due to a limitation of growth by diffusive delivering of $\mathrm{Si}$ atoms through bulk of catalyst, while the right one $R \leq R_{\max }$ taken into account for by the Gibbs-Thompson effect. It is clearly seen that there is a critical radius (indicated in Fig. 6 by

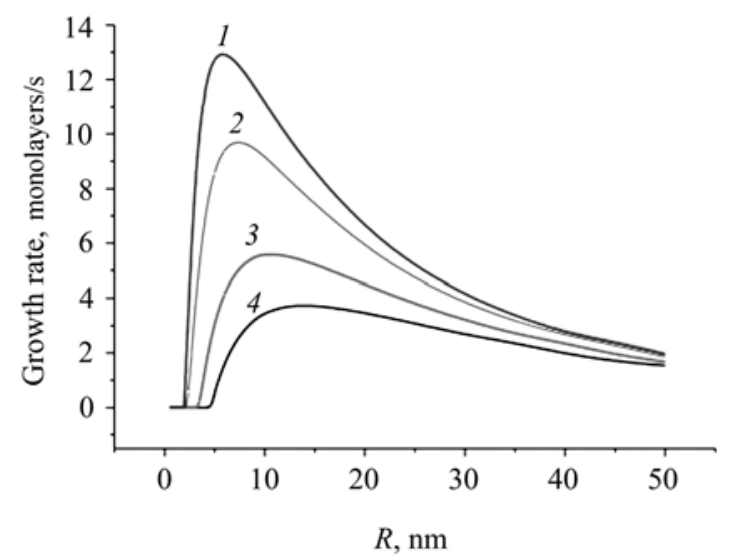

Fig. 5. Dependences of the wire growth rate versus $R$ at $T_{s}=913 \mathrm{~K}, \quad P_{\text {eq } 0}=20 \mathrm{mTorr}$ and different pressure of silane $P_{g}: 1-100,2-75,3-50,4-40$ mTorr. Bulk diffusion. 
$10 / R, \mathrm{~nm}^{-1}$
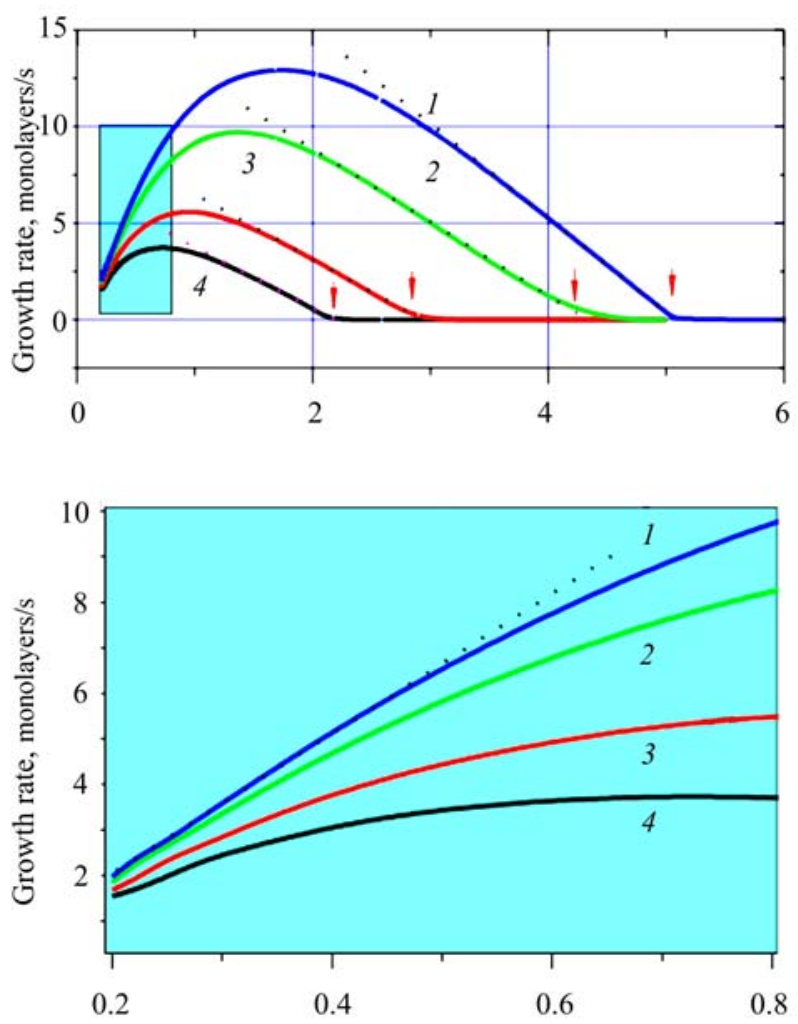

Fig. 6. Dependences of the wire growth rate versus $1 / R$ at $T_{s}=913 \mathrm{~K}, P_{\text {eq } 0}=20 \mathrm{mTorr}$ and different pressure of silane $P_{g}: 1-100,2-75,3-50,4-40$ mTorr. Bulk diffusion.

arrows), which depends on the partial pressure of silane, i.e., on oversaturation. Furthermore, the simulation shows that only nanowires with radii of restricted range of values can grow at certain growth conditions. This range is limited by the Gibbs-Thompson effect, on the one hand, and diffusion delivering the building blocks, on the other hand. This fundamental property of nanowire (nanoobject) growth is of great importance for controlling a main parameter of a nanoobject assembles like the radius scattering.

Dependences of the growth rate with a peak shown in Fig. 6 were experimentally observed in the works by A. Schetinin with coauthors [10, 27-29]. The left hand part of the curves was observed by Schubert with coauthors [30]. An effect by Gibbs-Thompson with the clear dependence of the critical radius on the value of oversaturation (the right hand part of the curves) was observed in the paper of E. Givargizov [32].

In contrast to the CVD growth considered in the current paper, the authors [30] used MBE technology. Nevertheless, we can also model the MBE growth and explain these results, because CVD growth includes all the processes involved into MBE growth. The left part of the dependences for large oversaturations in the gas phase is corresponding just to the case realized in [30].
In this case, the limiting stage of the wire growth is the diffusion through catalyst. It leads to inversely proportional dependence of the growth rate on the wire radius. At the same time, the growth rate does not depend on the growth time because diffusion direction does not change during the growth. Both these features were found experimentally in Ref. [30].

It is worth to note that an increase of the delivering rate of Si atoms toward the growing crystal, for instance, by increase of the diffusion coefficient or the substrate temperature, gives rise to a quite different dependence of the growth rate versus the wire radius. Fig. 8 shows such dependence calculated at a higher temperature and larger diffusion coefficient (surface diffusion). As can be seen instead of a sharp decrease of the growth rate for large radii (right hand part of the curves in Fig. 5), saturation of the growth rate is observed. An experimental conditions realized by J. Liu with coauthors [31] are likely to fit just such case. These authors have grown the silicon nanowires using the gas source molecular beam epitaxy (GS-MBE) and have found that neither axial nor radial growth rate depend on the wire radius.

An additional point to emphasize is that a character of growth rate dependence on the wire radius may be various. In the case studied by E. Givargizov, this dependence were approximated by the following formula (in our notation):

$\sqrt[n]{v_{h}} \propto \ln \frac{P_{g}}{P_{\text {eq } 0}}-\frac{2 \gamma_{c} \Omega_{c}}{k_{\mathrm{B}} T} \cdot \frac{1}{R}$,

with $n \approx 2$, whereas in our model we obtain a linear relation between $v_{h}$ and $1 / R$.

Indeed, according to Eqs (1)-(3) in the case when the growth is limited by a chemical reaction (it is the case of quick $\mathrm{Si}$ atom diffusion), for low oversaturation we should use the following simplified formula:

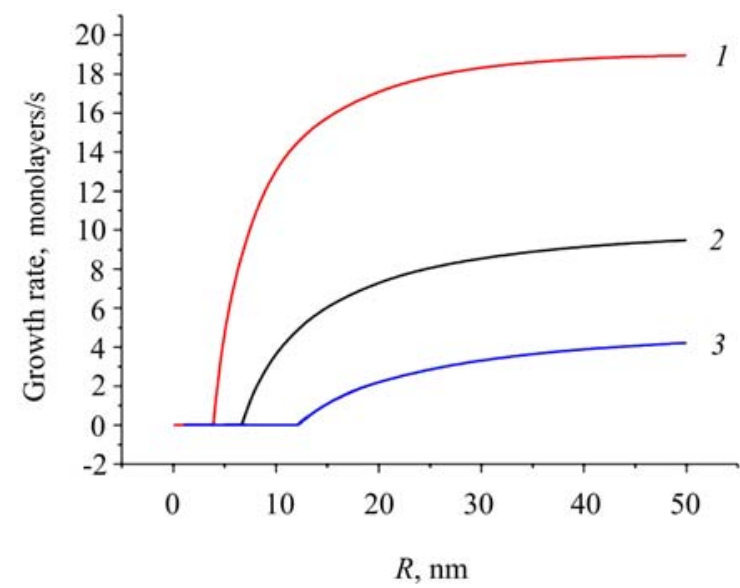

Fig. 7. Dependences of the wire growth rate versus $R$ at $T_{s}=1073 \mathrm{~K}, \quad P_{\text {eq } 0}=20 \mathrm{mTorr}$ and different pressure of silane $P_{g}: 1-40,2-30,3-25$ mTorr. Surface diffusion. 


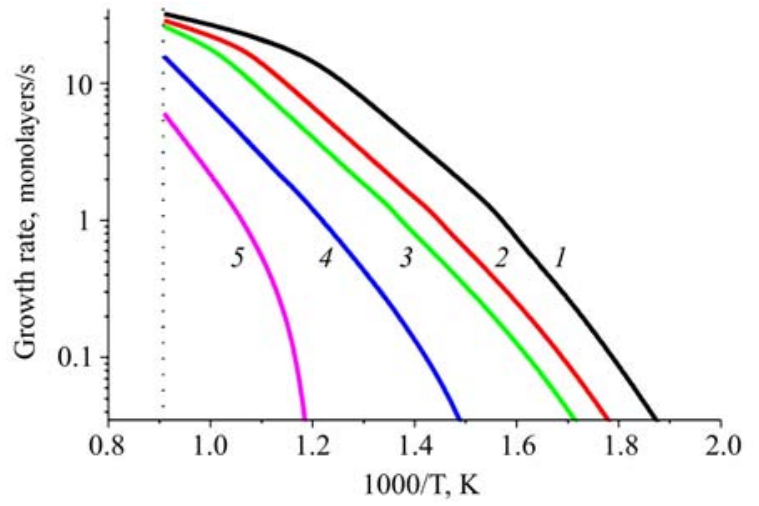

Fig. 8. Dependences of the wire growth rate versus $1 / T_{S}$ at $R=5 \mathrm{~nm}, P_{\text {eq0 }}=20 \mathrm{mTorr}$ and different pressure of silane $P_{g}: 1-200,2-100,3-75,4-50,5-40$ mTorr. Surface diffusion.

$$
\begin{aligned}
& v_{h}=2 \Omega_{c} v_{0} e^{-\frac{\Delta E_{a c}}{k_{\mathrm{B}} T}}\left(n_{g}-n_{\mathrm{eq} 0} e^{\frac{2 \gamma_{c} \Omega_{c}}{R k_{\mathrm{B}} T}}\right) \approx \\
& \approx 2 \Omega_{c} J_{0}\left(\xi-\frac{2 \gamma_{c} \Omega_{c}}{k_{\mathrm{B}} T} \cdot \frac{1}{R}\right), \\
& \xi=\frac{n_{g}}{n_{e q 0}}-1 \approx \ln \frac{n_{g}}{n_{\mathrm{eq} 0}}
\end{aligned}
$$

where $J_{0}=n_{\mathrm{eq} 0} v_{0} e^{-\frac{\Delta E_{a c}}{k_{\mathrm{B}} T}}$ and $\Delta E_{a c}=W_{a c}+\gamma_{c} \Omega_{c} K_{c} / 2$. So, we obtain the linear dependences of the growth rate both on the oversaturation and inverse radii likely to the found by simulation (Fig. 6). In the current model, we consider the growth of nanowires, and so restrict ourselves to a treatment of the growth based on onenucleation process at the catalyst-wire interface. The dependence of the growth rate defined by the formula (26) and found experimentally by E. Givargizov may arise from a large radius of the wires under investigation in that experiment and multiple two-dimensional nucleations at the interface. The latter usually gives rise to the dependence of the growth rate of the form (26) with $n \neq 1$, because a number of nuclei, radii of which equal to the critical one and which may grow simultaneously, depends in its turn on oversaturation degree $[25,26]$.

In summary of this discussion, it should be also pointed that there are experimental data [9] that obey the linear relation between these parameters at least for low oversaturations.

In Fig. 8, the temperature dependences of growth rate are presented. These results show that nanowires of the certain radius can grow only in a restricted region of the temperatures. An upper limit (indicated by the vertical doted line) is defined by the sharp increase of the growth rate on the substrate, which prevents from the onedimensional growth of wires, while the most low limit arises due to the Gibbs-Thomson effect. In a general case, in accordance with Eq. (26), the different effective activation energies are observed in the different temperature intervals.

Fig. 9 demonstrates dependences of the growth rate on the silane pressure for bulk (solid curves) and surface (doted curves) diffusion delivering $\mathrm{Si}$ atoms to the growing crystal. Both mechanisms give rise to saturation of the growth rate at high pressures while the upper limit of the rate is larger for larger temperatures. If we compare these dependences with Eqs (25) and (26), we can see some their resemblance as a whole with the logarithmic law $v_{h} \propto \ln ^{n}\left(P_{g} / P_{\text {eq } 0}\right) \propto(\Delta \mu)^{n}$, i.e., elongation growth rate likely depends only on chemical potential difference. However, in fact, for small $\xi$ we have $v_{h} \propto \xi$ in accordance with Eq. (26), and the whole dependence is found to be more complicate.

All the above simulations where we consider both the ways of building blocks delivering: surface and bulk diffusion, lead to the growth of nanowires in the form of solid cylinder, even if with a shell. The kinetics of such growth may be seen in Fig. 3 .

In the case when we take into account adsorption/ desorption and decomposition of silane on the sidewall (Fig. 1), crystal grows in a shape of solid cone and constitutes a heterostructure with a shell of the variable thickness along the big axis of the nanowire. The kinetics of this growth is presented in Fig. 4. The rate of a radial growth depends on the rate of the wire elongation and the

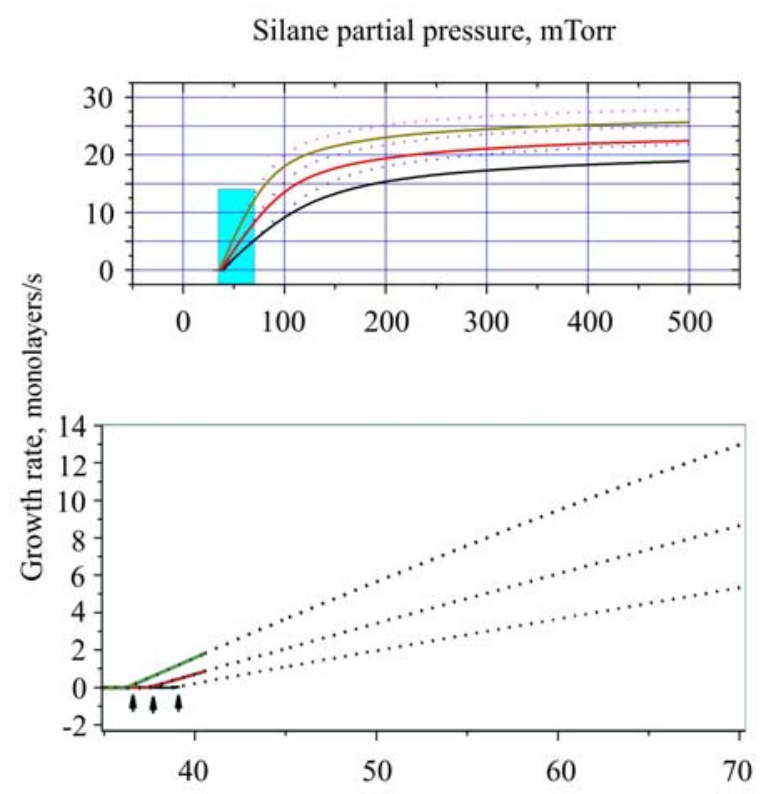

Fig. 9. Dependences of the wire growth rate versus pressure of silane $P_{g}$, wire radius $R=5 \mathrm{~nm}, P_{\mathrm{eq} 0}=20 \mathrm{mTorr}$ and different temperatures of the substrate $T_{S}$ : top-to-bottom 973, 923, $873 \mathrm{~K}$. Solid curves - bulk diffusion, doted curves surface diffusion. Arrows indicate the critical pressure of silane, excess of which leads to the wire growth. 
density of the growing wires on the substrate. So, it is evident that to suppress a cone-like growth of nanowires, one have to prevent the processes on the nanowire side. This may be done by using the selected dopants just as it was done by T. Kamins with coauthors in [34]. They introduce to the gas mixture $\left(\mathrm{H}_{2}+\mathrm{SiH}_{4}\right)$ additional component $\mathrm{HCl}$ that gives rise to the growth of nanowires in the form of a right cylinder.

Use of the dependences derived above can provide the method to control the process, that is, a technique to grow nanowires with the same length and a narrow scatter of radii. This goal may be achieved, e.g., (Figs 5 and 6) by suppressing the bulk and surface diffusion of $\mathrm{Si}$ adatoms using the specially selected dopants together with appropriate $T$ and $P$ manipulation.

The model predicts that under commonly used moderate oversaturation of the reactant gas, important characteristics, such as the axial and radial growth rates $v_{h}$ and $v_{r}$, depend primarily on the reaction rate at the surface of the catalyst or at the side of the nanowire and only partially on the gas-phase delivery of reactant. However, simulations showed that delivery of $\mathrm{Si}$ adatoms through the catalyst body or around its surface proves to be an important stage. In the case when this stage begins to limit the growth, we observe the dependences, which are typical for limiting by gas-phase delivery.

According to the simulations, the growth rates are also controlled by atomic hydrogen association and desorption; i.e., by removal of the by-products of silane decomposition from adsorption centers. We can conclude that the catalytic features of a catalyst particle can be caused not only by a low activation energy for adsorption $W_{a c}$ but also low activation barriers for atomic hydrogen association $\left(E_{\text {asc }}\right)$ and desorption $\left(E_{\text {des }}\right)$. Surface diffusion across the catalyst surface or interface becomes the limiting step, for example, under high oversaturation in the gas phase, or/and slow hydrogen desorption. In this case, hydrogen-containing species may occupy the noticeable part of diffusion sites on catalyst surface and some kind of "hydrogen-induced blockade" of silicon surface diffusion occurs. This effect is masked, however, when transfer between catalyst surface and the interface limits the delivery.

We note that two opposite processes (together with faceting [8]) determine the shape of the nanowire body: (i) lateral surface diffusion and (ii) surface drift induced by the surface free energy gradient. The latter may be caused, in turn, by the local inequality $\left(\gamma_{s} \cdot d K_{s} / d z+\right.$ $\left.+K_{s} \cdot d \gamma_{s} / d z\right) \neq 0$. Under steady-state conditions, the axial distribution of mobile adatoms on the nanowire side surface $\left(\gamma_{S}=\gamma_{\mathrm{Si}}=\right.$ const) is governed only by the local curvature:

$N_{a d}(z)=N_{0} \cdot \exp \left(-\frac{\gamma_{s} \Omega_{s} K_{s}(z)}{k_{\mathrm{B}} T}\right)$.

This effect, of course, is important at moderate temperatures. Therefore, nanowire surface regions with a constant curvature (parts of straight cylinder or sphere) will also have a uniform distribution of adatoms. As a result, nanowire growth in the radial direction should conserve the shape of the growing surface. Moreover, a necklace-like shape consisting of a set of spheres separated from each other by cylindrical segments with $R_{\text {cyl }}=R_{\text {sph }} / 2$ has a constant curvature, and therefore, at moderate temperatures is also a stable shape in good agreement with the experiment [4]. In some cases, the elastic part of the total free energy should be considered; that may explain the tube-like microobjects [33]. We conclude according to Eq. (27) that a conical shape is unstable due to the curvature gradient along the axial direction. The tendency to form this shape may be caused primarily by gas adsorption and reaction on the side of the wire. The portion of the nanowire near the bottom will have a larger radius than the upper portion parts because it is formed earlier and is, therefore, exposed to the incident gas for a longer period of time [34]. The flux of adatoms from the nanowire tip (highest curvature $\mathrm{K}$ ) to its bottom (lowest $\mathrm{K}$ ) also increases the tendency to form a conical shape. In addition, the theory predicts that the telescopic shape (with sections of different, constant diameter) sometimes observed after heat treatments [35] is more stable than a cylinder of the same volume.

The approach considered in this paper allows us to predict the formation of core/shell nanostructure with solid, compact shell. However, it is unlikely to be applicable to the explanation of the nano-cellular structure of the shell formed on the sides of nanowires grown in closed tube [36, 37]. The nanocells observed in those experiments share their facets and have hexagon or pentagon cross-section. Dividing the total energy into surface and bulk parts is questionable because of nanoscale of the cells; in this case, ab initio calculations of the structure are more appropriate.

\section{Conclusions}

A phenomenological model has been developed for silicon nanowire growth on silicon substrates by CVD using $\mathrm{SiH}_{4}+\mathrm{H}_{2}$ mediated by catalyst particles. The dependence of the nanowire growth kinetics on radius, substrate temperature, and partial pressure of Si containning gas has been demonstrated. Good qualitative agreement between the simulations and published experimenttal data was obtained. The peculiarities of the nanowire growth are discussed, and possible methods to control the process and shape transformation are considered.

\section{Acknowledgements}

This work was performed in the framework of CRDF Project \# UE-5001-KV-03. The authors wish to express their sincere gratitude to Prof. P.M. Tomchuk (Institute of Physics, National Academy of Sciences of Ukraine) for helpful discussions. The authors also thank Dr. S. Sharma and Dr. R. Stanley Williams of Hewlett-

(C) 2005, V. Lashkaryov Institute of Semiconductor Physics, National Academy of Sciences of Ukraine 
Packard for providing experimental data and for useful discussions.

\section{References}

1. T.I. Kamins, MRS Fall Meeting Abstracts, November 29 - December 3, 2004, p. 14.

2. T.I. Kamins, R.S. Williams, D.P. Basile, T. Hesjedal, J.S. Harris // J. Appl. Phys. 89, p. 1008 (2001).

3. D. Appell // Nature 419, p. 553 (2002).

4. E.I. Givargizov, Growth of filament-like and platelet crystals from vapor, Moscow, Nauka (1977) (in Russian);

5. D.N. McIlroy, D.Zhang, Y. Kranov and M. Grant Morton // Appl. Phys. Lett. 79, p. 1540 (2001).

6. D.N. McIlroy, A. Alkhateeb, D. Zang et al. // J. Phys.: Condens. Matter 16, p. R415 (2004).

7. A.I. Klimovskaya, E. G. Gule, I.V. Prokopenko, Proceedings of $17^{\text {th }}$ Quantum Electronics Conference (MIEL 2000), Nis, Yugoslavia.

8. N. Combe, P. Jensen, A. Pimpinelli // Phys. Rev. Lett. 85, p. 110 (2000); W.W. Mullins and G.S. Rohrer // J. Amer. Ceram. Soc. 83, p. 214 (2000).

9. Yukichi Tatsumi, Mitsuji Hirata and Mikio Shugi // Jpn J. Appl. Phys. 18, p. 2199 (1979).

10. A.A. Schetinin, O.D. Kosenkov, A.V. Gilyarovskii, E.E. Popova // Neorganicheskie materialy 25, p. 1237 (1989) (in Russian).

11. F. Hirose // J. Cryst. Growth 179, p. 108 (1997).

12. A. Frank-Kamenetskii, Diffusion and heat transfer in chemical kinetics. Moscow, Nauka (1987) (in Russian).

13. E.M. Livshitz, L.P. Pitaevskii, Physical kinetics. Vol. $X$. Theoretical physics. Moscow, Nauka (1979) (in Russian).

14. D.V. Sivukhin, General course of physics. Vol II. Thermodynamics and molecular physics. Nauka, Moscow (1990) (in Russian).

15. G.A. Bird, Molecular gas dynamics. Oxford, Clarendon Press (1976).

16. C.W. Christian, The theory of transformation in metals and alloys. Part 1. Equilibrium and general kinetic theory. Oxford, Pergamon (1975).

17. S. Senkander, I. Esfandyary, G. Hobler // J. Appl. Phys. 78, p. 6 (1995).

18. B.Ya. Lyubov, Diffusion processes in inhomogeneous solids, Nauka, Moscow (1981) (in Russian).

19. J. Bloem // Pure and Apply Chemistry 50, p. 435 (1978).

20. W. Moench, Semiconductor surfaces and interfaces. Springer-Verlag, Berlin, Heidelberg (1993).

21. In the general case of multicomponent surface diffusion, the term $D_{k} \Theta_{k}$ can be represented in the following form: $D_{0} \nabla\left\{\left[1-\Sigma \Theta_{k}\right] \nabla \Theta_{k}+\Theta_{k} \Sigma \nabla \Theta_{k}\right\}$, where $D_{0}$ is the one-component diffusion coefficient and $\Theta_{k}$ is the fractional coverage of the $k$-th adatoms. The commonly used expression for this term $D_{0} \Delta \Theta_{k}$ is valid only for a small difference in the one-component diffusion coefficients and/or for small coverages of different adatoms. But here we do not solve continual diffusion equation.

22. R. Stratonovich // Non-linear non-equilibrium thermodynamics. Nauka, Moscow (1985)(in Russian).

23. P. Blaha, K. Schwarz, G.K.H. Madsen, D. Kvasnicka and J. Luitz, WIEN2k, An Augmented Plane Wave+Local Orbitals Program for Calculating Crystal Properties (Karlheinz Schwarz, Techn. Universität Wien, Austria), 2001. ISBN 3-9501031-1-2.

24. G.A.D. Briggs, D.P. Basile, G. Medeiros-Ribeiro, T.I. Kamins, D.A.A. Ohlberg, and R. Stanley Williams // Surf. Sci. 457, p. 147 (2000).

25 . Probability of one or multiple nucleation depends on a size of interface, i.e. radius of a wire, oversaturation, and the critical radius of the nucleus. If a rate of creation of nucleus is smaller than a rate of step propagation toward the edge of interface, than the wire grows by the mechanism of one nucleation. One nucleation is a likely pathway for growth of nanowires.

26. A.A. Chernov // Kristallografia 16, p. 842 (1971) (in Russian).

27. A.A. Schetinin, O.D. Kozenkov, A.I. Dunajev // Zhurnal Tekhnicheskoy Fiziki 53, p.1416 (1983) (in Russian).

28. B.M. Darinskii, O.D. Kozenkov, A.A. Schetinin // Izvestiya Vuzov MV\& SSO, Fizika Tverdogo Tela 12, p. 18 (1986).

29. A.A. Schetinin, L.I. Bubnov, O.D. Kozenkov, A.F. Tatarenkov // Neorganicheskie materialy 23, p. 1589 (1987) (in Russian).

30. L. Schubert, P. Werner, N.D. Zakharov et al. // Appl. Phys. Lett. 84, p. 4968 (2004).

31. J.L. Liu, S.J. Cai, G.L. Jin, and K.L. Wang // Electrochemical and Solid-State Letters 1, p. 188 (1998).

32. E.I. Givargizov // J. Crystal Growth 31, p. 20 (1975).

33. N.I. Vitrichovskii, B.I. Lev, P.M. Tomchuk // Ukr. Phys. Journ. 33, p. 1713 (1988).

34. S. Sharma, T.I. Kamins, and R.S. Williams // J. Crystal Growth 267, p. 613 (2004).

35. T.I. Kamins, X. Li, and R.S. Williams // Appl. Phys. Lett. 82, p. 263 (2003).

36. A.I. Klimovskaya, I.V. Prokopenko and I.P. Ostrovskii // J. Phys.: Condens. Matter 13, p. 5923 (2001).

37. A.I. Klimovskaya, I.V. Prokopenko, S.V. Svechnikov et al. // J. Phys.: Condens. Matter 14, p. 1735 (2002). 